\title{
Mentorship at Distance: A new initiative of the Journal of Nuclear Cardiology
}

\author{
Frans J. Th. Wackers, MD, PhD, FACC, FASNC, a and Ami E. Iskandrian, MD, \\ MACC, FASNC ${ }^{\mathrm{b}}$ \\ a Section of Cardiovascular Medicine, Yale University School of Medicine, New Haven, CT \\ ${ }^{b}$ University of Alabama at Birmingham, Birmingham, AL
}

Received Mar 9, 2015; accepted Mar 9, 2015

doi: 10.1007/s12350-015-0117-4

The Journal of Nuclear Cardiology embarked recently on a new project, "Mentorship at Distance.", This was envisioned as a service to assist authors and scientists, whose native language is not English, with the publication of their research.

The Journal of Nuclear Cardiology is an Englishlanguage journal that aims to reach a worldwide international audience; more than $50 \%$ of the papers submitted to the Journal in 2014 were from outside the United States. This is also true for most, if not all, major cardiology journals. However, only $5.4 \%$ of the world's population (360 millions in 2007) is native English speaking, although a comparable number (400 millions) speaks English as a second language. English is recognized as the lingua franca in science and business.

Speaking a foreign language is one thing, but writing grammatically correct English is something else. Furthermore, writing in English about science and research requires yet another skill. Thus, non-native speakers may be at some disadvantage when attempting to publish their scientific research in the major Englishlanguage professional journals.

For speakers of Western languages, it is relatively easy to learn English as a second language, because the (Indo-) European languages are loosely related through grammar and vocabulary. However, for speakers from other language families, it may be substantially more difficult. Not only may the syntax of a language be totally different, but also certain concepts that can be expressed in one language may be impossible or difficult to articulate in another language.

Reprint requests: Frans J. Th. Wackers, MD, PhD, FACC, FASNC, Section of Cardiovascular Medicine, Yale University School of Medicine, New Haven, CT; frans.wackers@yale.edu J Nucl Cardiol 2015;22:403-4.

$1071-3581 / \$ 34.00$

Copyright (C) 2015 American Society of Nuclear Cardiology.
All manuscripts submitted to this Journal undergo peer review by experts in the field of cardiovascular imaging. The task of a reviewer is not only to judge the scientific merits of a manuscript, but also to critique and make suggestions on how a manuscript could be improved. The presentation of data may need further clarification, clearer focus, and/or additional analysis. All of us, even the most experienced authors, have felt the "pain" of a thorough review. But, we also have benefited from this valued process and we acknowledge that in the end our work was better because of it. It is easy and regrettably done too often, to reject a manuscript just because of linguistic problems. For cardiovascular imaging, this may mean that a sizable number of new investigators are denied publication rights, and with it the impetus to submit future work and grow academically.

Sometimes reviewers of the Journal identify manuscripts from abroad that appear to have potential, but that are not acceptable in its current form. The data may seem to be sound and valuable, but the "packaging", is poor. Such manuscripts may benefit from substantial editing and rewriting. The problem does not always consist of language issues, but can encompass also other shortcomings, such as failure to state a clear purpose of study, improper analysis and presentation of data, and suboptimal scholarly discussion. The Journal now offers authors of selected manuscripts the opportunity to work under the mentorship of experienced authors to improve their manuscript. The reality is that reviewers and editors do not have the time to do the mentoring on routine basis and therefore an independent committee was deemed necessary.

In 2014, the Journal established a Committee called "Mentorship at Distance"' and one of the authors (FW) is the Chair. Several Board members of the Journal and Associate and Guest editors volunteered to serve on that committee.

Thus far, several manuscripts have gone through the "Mentorship at Distance"' program. This turned out to 
be an interesting and rewarding experience for both the authors and the mentor. As was to be expected, some manuscripts were at the end not salvageable, but others improved sufficiently and were accepted for publication.

The process is at present time as follows: after the regular peer review, the editors discuss the merits of the manuscript and the potential for improvement by involving the Mentorship at Distance Committee. If this is agreed upon, the authors and the Chair of the committee are notified. So far, the response has been extremely positive and frankly beyond our expectation.

Once the authors have accepted the proposed mentorship, the mentor and senior author enter into an intense communication, often by daily email exchanges. The mentor is provided with a copy of the original or revised manuscript, as well as all reviews. It is clear that a language barrier may make the formulation of a research question, the writing of a well-organized discussion and succinct conclusion, very challenging. The mentor's role is not only to polish the language, but also to help in reanalyzing and reorganizing the data. Manuscripts sometimes suffer from too much and not essential information and analyses. A fresh look may be all that is needed to bring out the merits of a study.

In order to gain a better understanding of the research, the mentor may request additional data and clarifications. This is the fun part for the mentor: perusing the raw material, extracting what is essential, dismissing what is not, and finally coming to a logical conclusion that is supported by data. Once the manuscript is stripped from vagaries and redundancies and the line of reasoning and final conclusion is reassessed by mutual agreement, the rewriting of the manuscript can start. For efficiency reasons, it is inevitable that this will be done for a large part by the mentor him- or herself. The mentor will rewrite the manuscript anew, while preserving as much as possible edited parts of the original manuscript.

Manuscripts that accomplished publication through this mentoring process will be identified in the Journal under the acknowledgments. It is important to understand that the responsibility for the authenticity and validity of the data rest solely with the authors and not with either the mentor or the Journal. The authors understand that acceptance of the edited manuscript is not guaranteed. The re-submitted manuscript will be reviewed again rigorously, but at least it now has a second chance that it did not have before the process. The mentor him-/herself has no self-interest in this process, other than the satisfaction of mentoring authors who lack experience in scientific writing in English. Many of us certainly can relate to this by remembering our own experiences of writing our first research papers, or when we started editing the research work by our trainees!

The Mentorship at Distance program of the Journal appears to be off to a good start. We are confident that it will become a very worthwhile and rewarding service to our authors from abroad. We anticipate the demand for the Mentorship at Distance program will grow and the Chair of the Committee will call upon other members to help out. We also would not be surprised if other journals would follow this initiative, as the concept is unique and important. 